\title{
Intensive home treatment, admission rates and use of mental health legislation
}

\author{
Naida F. Forbes, ${ }^{1}$ Helen T. Cash, ${ }^{2}$ Stephen M. Lawrie ${ }^{3}$
}

The Psychiatrist (2010), 34, 522-524, doi: 10.1192/pb.bp.109.027417

${ }^{1}$ Royal Edinburgh Hospital, Edinburgh; ${ }^{2}$ South East Scotland Deanery;

${ }^{3}$ University of Edinburgh, Edinburgh

Correspondence to Naida F. Forbes (naida.forbes@nhslothian.scot.nhs.uk)

\begin{abstract}
Aims and method We examined the local impact of introducing a home treatment team on the use of in-patient psychiatric resources and rates of detention under the Mental Health (Care and Treatment) (Scotland) Act 2003.
\end{abstract}

Results Rates of admission to hospital and duration of hospital stay were unchanged. However, there was an increase in episodes of detention in the year following the team's introduction.

Clinical implications Offering home treatment as an alternative to in-patient care may be associated with an increase in compulsory treatment. If true, this is incompatible with the 'least restrictive alternative' principle of the recently revised mental health legislation.

Declaration of interest N.F.F. was until recently employed by Midlothian Community Health Partnership.
Crisis resolution and home treatment teams have been widely introduced as directed by the National Health Service Plan. ${ }^{1}$ There is a growing body of robust evidence to demonstrate that offering intensive home treatment reduces the need for in-patient care, ${ }^{2,3}$ although relatively little has been published about the effect of these teams on rates of compulsory admission to hospital under mental health legislation.

Additional drivers to develop services offering alternatives to hospital care are reflected in both the Mental Health (Care and Treatment) (Scotland) Act 2003 and the 2007 amendments to the Mental Health Act 1983 for England and Wales. Central to both pieces of legislation is a set of fundamental, guiding principles. One of these principles is that of the least restrictive alternative: 'People taking action without a patient's consent must attempt to keep to a minimum the restrictions they impose on the patient's liberty'. In addition, both acts call for any compulsory intervention to be of demonstrable benefit to the individual.

We set out to examine the effect of introducing a home treatment team on the rates of in-patient care and compulsory treatment in the local area. Midlothian is a semi-rural area with a total population of approximately 82000 , lying immediately south of the city of Edinburgh. An extensive redesign of its adult mental health service was recently launched, in partnership between health service, local authority and non-statutory organisations, and following wide consultation with service users and carers. Central to this redesign was the closure of the local 19-bed adult in-patient ward, replaced by an allocation of 8 beds at the Royal Edinburgh Hospital, 10 miles away. To support this move, adult community mental health services were reconfigured with the launch of an intensive home treatment team (IHTT). The aims of the IHTT were to support the planned reduction in bed numbers by providing a robust alternative to hospital admission for some individuals and by facilitating early discharge from hospital. The IHTT operated between $08.00 \mathrm{~h}$ and $24.00 \mathrm{~h} 7$ days a week, and was staffed by a multidisciplinary team of mental health nurses, a part-time staff grade psychiatrist, a support worker and an occupational therapist, with ready access to social work, psychology and physiotherapy. Potential admissions were discussed with and frequently assessed by IHTT during their working hours. Consultant responsibility for patient care was allocated to one of the three consultant adult psychiatrists based on individuals' general practitioners' (GP) practices, with the same consultant retaining responsibility whether the person was in hospital, an out-patient or under the care of the IHTT to maintain continuity of care. The closure of the local in-patient unit, and the launch of the IHTT, both occurred on 30 April 2007. We set out to examine the effectiveness of the IHTT in providing a robust alternative to in-patient care by measuring the number of admissions, length of hospital stay and average number of beds occupied per day over the first year of its operation (1 May 2007 to 30 April 2008). For comparison, we measured the same variables over the preceding year (1 May 2006 to 30 April 2007). In addition, we surveyed the rate of detentions under the Mental Health (Care and Treatment) (Scotland) Act 2003 for both periods.

We expected that the IHTT would provide an alternative to hospital care for a number of individuals, which should be reflected in admission rates, length of stay, 
bed occupancy and possibly in detention rates. We also hypothesised that, as some people who would previously have been offered admission could now be offered the alternative of IHTT care, those who did require admission may be more unwell and that this may be reflected in a higher proportion of detained patients in the year following redesign. We expected overall rates of detention to remain the same.

\section{Method}

We collected data on admissions to general adult psychiatry beds for adults aged 18-64, registered with a GP in Midlothian, between 1 May 2007 and 30 April 2008. We examined the same data for the period 1 May 2006 to 30 April 2007 for comparison. This information was obtained from the electronic database where all admissions and discharges are routinely recorded, and was double-checked against manual records kept by staff. We recorded the number of admissions and the number of nights spent in hospital during each admission. For both time periods, we added up the total number of bed nights occupied and divided this by the number of nights in the period to calculate average bed occupancy.

We also collected data describing detentions under the Mental Health (Care and Treatment) (Scotland) Act 2003 during both time periods, where adults aged 18-64 with a GP in Midlothian were detained under the care of general adult psychiatrists. This information was obtained from social work colleagues in the mental health team who coordinate all Health (Care and Treatment) (Scotland) Act 2003 assessments for people from Midlothian and doublechecked against admission records and patients' case notes where necessary.

\section{Results}

Admission rates did not differ markedly across the two time periods, although a larger proportion of individuals were detained at some point during their admission in the 20072008 period (Fig.1). For both time periods, the median length of stay in hospital was 9 nights; the mean length of stay was 18 nights in the 2006-2007 period and 19 nights in the 2007-2008 period. The average number of beds occupied per night dropped from 9 in the 2006-2007 period to 6 in the 2007-2008 period. This may be partly explained by the presence of 16 people with prolonged stays in hospital at the start of the 2006-2007 period, versus only 2 individuals in hospital at the start of the corresponding period in 2007-2008. In the 2007-2008 period, IHTT received 237 referrals, of which 133 were taken on for intensive home treatment.

As anticipated, the proportion of compulsory admissions increased, but we also found an unexpected increase in the absolute number of people subject to detention under the Mental Health (Care and Treatment) (Scotland) Act 2003. Upon examining the data further, we found marked increases in the rates of all civil compulsory orders (both hospital- and community-based) in the year following redesign (Fig. 2).

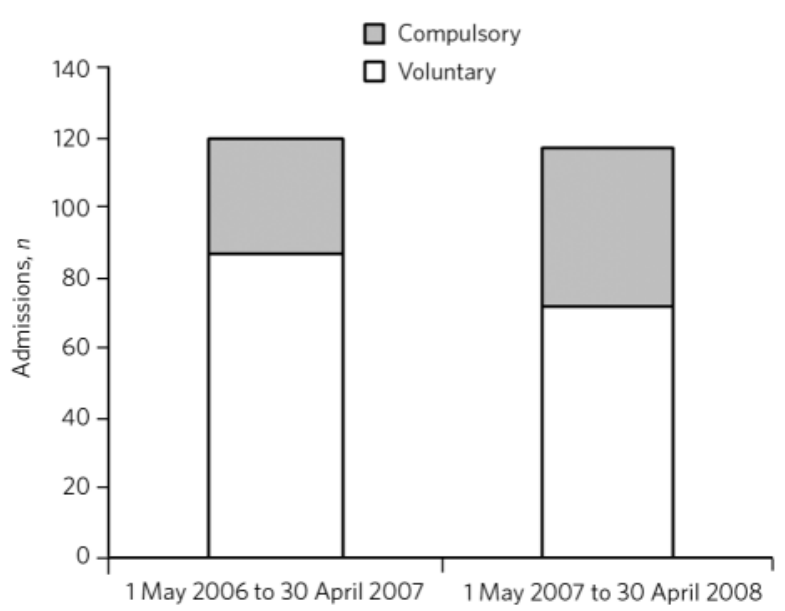

Fig 1 Impact of Midlothian mental health service redesign on admission rates to general adult psychiatry beds.

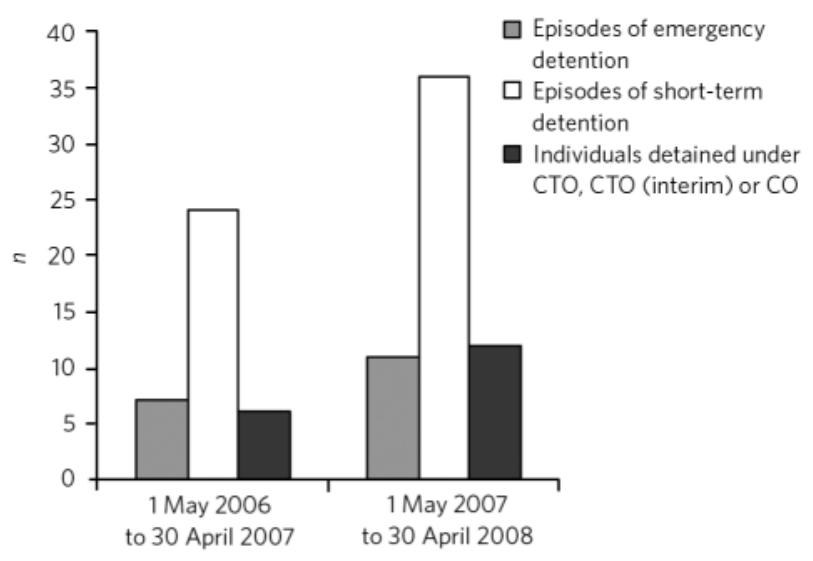

Fig 2 Impact of Midlothian mental health service redesign on Mental Health (Care and Treatment) (Scotland) Act 2003 activity for general adult psychiatry.

CTO, compulsory treatment orders; CO, compulsion order.

\section{Discussion}

Contrary to our expectations, the service redesign and introduction of the IHTT in Midlothian has not been associated with reduced admission numbers or length of hospital stay. The introduction of the IHTT was associated with a reduction in average bed occupancy, which may be explained by the presence of a cohort of in-patients, with long hospital stays at the start of the 2006-2007 period.

The most noticeable finding from this study has been the increase in the numbers of individuals admitted to hospital on a compulsory basis during the 2007-2008 period, associated with the IHTT's introduction. Our data show a convincing rise in admissions under all three of the civil compulsory orders: i.e. short-term detention certificates (completed by an approved medical practitioner with mental health officer consent, authorising up to 28 days in hospital for assessment or treatment), emergency detention certificates (completed by any registered doctor, usually with mental health officer consent, and generally used out of hours when there was no provision for immediate approved medical practitioner availability) and more 
strikingly a twofold increase in compulsory treatment orders. An application for the compulsory treatment order is made by the mental health officer, supported by two medical reports, and heard before the Mental Health Tribunal for Scotland. If granted, a compulsory treatment order can authorise medical treatment and other powers for up to 6 months initially, either in hospital or in the community. Keown et $a l^{5}$ identified a similar trend after the establishment of a crisis and assertive outreach team in Newcastle, but few other studies have replicated this. In contrast to those findings and our present findings, Johnson's group ${ }^{2,6}$ reported no significant change in detention rates associated with crisis care.

We considered several possible explanations for the increase in compulsory admissions following the introduction of the IHTT. Our findings could be a consequence solely of the natural fluctuation in rates and severity of acute mental illness measured over a relatively short time period. An alternative explanation may be that individuals who would previously have been admitted to their local hospital on a voluntary basis were not willing to consent to admission to the new unit - however, this was not our impression from our knowledge of the individuals and the circumstances surrounding their admissions.

On the other hand, it may be that this new team had a low threshold for accepting risk and was more likely to consider use of the Mental Health (Care and Treatment) (Scotland) Act 2003 to manage risks associated with clinical situations. Certainly, the introduction of formal riskassessment procedures for every patient seen by the team resulted in a heightened awareness of risk. The IHTT also had access to more background information about individuals when performing emergency assessments than had previously been available, a factor previously shown to increase likelihood of admission. ${ }^{7}$

The team was reviewing people regularly (up to twice daily for the most unwell individuals on their case-load), and these repeated assessments may have increased the chances of encountering a situation where the combined clinical presentation and risks meant that detention was appropriate. Assessing people in the home setting enables carers to voice their views and concerns more freely, which can prove a valuable addition to the risk assessment.

We wonder also whether thresholds for assessing longterm risk of non-adherence and relapse following discharge are changing. Our observed increase in use of longer-term detentions (compulsory treatment orders) may relate either to the activity of the IHTT or to the reduction in bed numbers. It is possible that clinicians are more inclined to use community detention powers as a means of pre-empting revolving-door admissions in a climate where in-patient resources are more scarce. When using the mental health legislation, we must be mindful that this is motivated by the likely benefit to the service user, rather than the constraints of service provision.

\section{Implications}

We hypothesised that the availability of intensive home treatment as an alternative to admission may have led to a reduction in admission rates, length of stay and use of compulsory powers, in accordance with the 'least restrictive alternative' principle. However, we found an association between the introduction of an IHTT service and an increased use of mental health legislation, without notable change in rates of admission to in-patient care or length of stay in hospital. The national trend for resources to shift from in-patient facilities towards IHTT models is accelerating. We suggest that the success of this strategy should be evaluated not only by collating admission and bed usage statistics, but also monitoring rates of mental health legislation usage.

\section{About the authors}

Dr Naida F. Forbes is a consultant psychiatrist at Royal Edinburgh Hospital, Edinburgh. Dr Helen T. Cash is an ST6 in psychiatry in the South East Scotland Deanery. Professor Stephen M. Lawrie is a professor of psychiatry and neuroimaging at the University of Edinburgh, Edinburgh.

\section{Acknowledgements}

We are grateful to Mrs Susan Heggie, Mr Bruce Hadden, Mrs Sheila Blyth, Mrs Anne Hall, Mrs Marilyn Robertson and Dr Andrew Mclntosh for their assistance.

\section{References}

1 Department of Health. The NHS Plan: A Plan for Investment, A Plan for Reform. Department of Health, 2000

2 Johnson S, Nolan F, Hoult J, White IR, Bebbington P, Sandor A, et al. Outcomes of crises before and after introduction of a crisis resolution team. Br J Psychiatry 2005; 187: 68-75.

3 Glover G, Arts G, Babu KS. Crisis resolution/home treatment teams and psychiatric admission rates in England. Br J Psychiatry 2006; 189: 441-5.

4 Department of Health. Mental Health Act 1983: Code of Practice (2008 Revised). Department of Health, 2008.

5 Keown P, Tacchi MJ, Niemiec S, Hughes J. Changes to mental healthcare for working age adults: impact of a crisis team and an assertive outreach team. Psychiatr Bull 2007; 31: 288-92.

6 Johnson S, Nolan F, Pilling S, Sandor A, Hoult J, McKenzie N, et al. Randomised controlled trial of acute mental health care by a crisis resolution team: the North Islington crisis study. BMJ 2005; 331: 559602.

7 Shajahan P, Agnew T. Availability of patient records and psychiatric admission rate. Psychiatr Bull 2006; 30: 449-51. 